\title{
ON TWO LEMMAS OF BROWN AND SHEPP HAVING APPLICATION TO SUM SETS AND FRACTALS, III
}

\author{
N. ELEZOVIĆ 1 , M. MATIĆ ${ }^{2}$, C. E. M. PEARCE ${ }^{3}$ and J. PEČARIĆ ${ }^{3}$
}

(Received 22 August 1997)

\begin{abstract}
We improve some results of [17], which relate to key tools given in [7] for establishing canonical inequalities used in the analysis of sum sets and fractals.
\end{abstract}

\section{Introduction}

In recent years a number of striking results have been derived for probability measures of certain sum sets involving fractals (see, for example, $[2,4,5,8,9,13])$. By the algebraic sum set $E+F$ of two sets $E, F$ we signify the set

$$
E+F=\{x+y: x \in E, y \in F\} .
$$

The earliest and simplest of these results, established in [5], is the following.

THEOREM A. Suppose m denotes Lebesgue measure and $\mu_{c}$ Cantor-Lebesgue measure, that is, the uniform distribution on the Cantor subset $C$ of $[0,1]$ formed by repeated removal of middle thirds. If $E, F$ are Borel subsets of $[0,1]$, then

$$
m(E+F) \geq 2 \mu_{c}(E)^{\alpha} \mu_{c}(F)^{\alpha},
$$

where $\alpha=\log 3 / \log 4$.

These results depend on novel inequalities involving nonintegral powers of real numbers. Thus Theorem A uses the inequality

$$
x^{\alpha} y^{\alpha}+\max \left\{x^{\alpha}(1-y)^{\alpha}, y^{\alpha}(1-x)^{\alpha}\right\}+(1-x)^{\alpha}(1-y)^{\alpha} \geq 1 \quad(0 \leq x, y \leq 1)
$$

${ }^{1}$ Department of Applied Mathematics, Faculty of Electrical Engineering and Computing, Unska 3, 10000 Zagreb, Croatia

${ }^{2}$ Department of Mathematics, FESB, R. Boškovića B.B., 21000 Split, Croatia

${ }^{3}$ Department of Applied Mathematics, The University of Adelaide, SA 5005, Australia

(C) Australian Mathematical Society 2000, Serial-fee code 0334-2700/00 
established by Woodall [19].

Woodall and subsequently Hajela and Seymour [10] derived a variety of interesting results in combinatorial geometry from the latter inequality, which is therefore of some interest in its own right. The history of these results and related ideas pertaining to a multivariate extension of $(1.1)$ (see $[4,12]$ ) is quite colourful. A brief account is given by Brown [2].

Inequalities like (1.1) may be shown to depend on canonical univariate inequalities (see Brown [2,3]). Thus (1.1) can be deduced from the following result.

PROPOSITION B. Suppose that $s, t \geq 1$ and $s^{-1}+t^{-1}=\log 3 / \log 2$. Then

$$
1+x+x^{2} \geq\left(1+x^{s}\right)^{1 / s}\left(1+x^{t}\right)^{1 / t}
$$

for all for $0 \leq x \leq 1$ if and only if $3(s+t) \leq 8$.

These inequalities are central. For example, (1.2) also leads to more general multivariate inequalities than (1.1) and consequent new measure-theoretic inequalities. A comprehensive account is given in [6].

Establishing the canonical univariate inequalities can be quite tricky and some effort has been put into sharpening techniques for their derivation (see [7, 11, 14-17] and most recently [1]). The 'two lemmas' of the title are special cases of the two parts of Theorem 3.2 below, the earliest versions of which are due to Brown and Shepp [7] and have influenced further work in the area.

This paper is a continuation of [17], from which we take the terminology and notation. We are able to improve the main theorems of [17], due to improvements in the auxiliary results and lemmas. In Section 2 we give a strengthened form of the main lemma underlying the results of [1]. Improved forms of several existing theorems follow immediately and are given in Section 3.

\section{Key results}

In [1] the following theorem is proved.

THEOREM C. Let $p_{i}(i=1, \ldots, n ; n \geq 2)$ be positive real numbers with $\sum_{i=1}^{n} p_{i}=1$. Then for all real numbers $a_{1}, \ldots, a_{n}$ we have

$$
\max _{1 \leq i \leq n} a_{i}-\sum_{i=1}^{n} p_{i} a_{i} \geq \frac{p}{n-1} \sum_{1 \leq i<j \leq n}\left(\sqrt{\left|a_{i}\right|}-\sqrt{\left|a_{j}\right|}\right)^{2},
$$

where

$$
p=\min _{1 \leq i \leq n} p_{i}
$$


The proof of this theorem is rather complicated, especially for the case when the quantities $a_{i}$ are not all of the same sign.

We shall give a very simple proof of the following improvement of this theorem.

THEOREM 2.1. Let $p_{i}(i=1, \ldots, n ; n \geq 2)$ be positive real numbers with $\sum_{i=1}^{n} p_{i}=1$ and $a_{1}, \ldots, a_{n}$ real numbers. Suppose

$$
a_{i^{\prime}}=\max _{1 \leq i \leq n} a_{i}
$$

Then we have

$$
a_{i^{\prime}}-\sum_{i=1}^{n} p_{i} a_{i} \geq \frac{p^{\prime}}{n-1} \sum_{1 \leq i<j \leq n}\left|a_{i}-a_{j}\right|
$$

where $p^{\prime}=\min _{i \neq i^{\prime}} p_{i}$.

PROOF. Let $b_{n} \leq b_{n-1} \leq \cdots \leq b_{1}$ be a permutation of $a_{1}, \ldots, a_{n}$ and $r_{1}, \ldots, r_{n}$ the corresponding permutation of $p_{1}, \ldots, p_{n}$. Then

$$
a_{i^{\prime}}-\sum_{j=1}^{n} p_{j} a_{j}=b_{1}-\sum_{j=1}^{n} r_{j} b_{j}=\sum_{j=2}^{n} r_{j}\left(b_{1}-b_{j}\right) .
$$

For each $i \in\{1, \ldots, n-1\}$ we have

$$
\sum_{j=2}^{n} r_{j}\left(b_{1}-b_{j}\right) \geq \sum_{j=i+1}^{n} r_{j}\left(b_{1}-b_{j}\right) \geq \sum_{j=i+1}^{n} r_{j}\left(b_{i}-b_{j}\right) \geq p^{\prime} \sum_{j=i+1}^{n}\left(b_{i}-b_{j}\right) .
$$

Hence

$$
\begin{aligned}
a_{i^{\prime}}-\sum_{j=1}^{n} p_{j} a_{j} & =\frac{1}{n-1} \sum_{i=1}^{n-1} \sum_{j=2}^{n} r_{j}\left(b_{1}-b_{j}\right) \\
& \geq \frac{1}{n-1} p^{\prime} \sum_{i=1}^{n-1} \sum_{j=i+1}^{n}\left(b_{i}-b_{j}\right) \\
& =\frac{p^{\prime}}{n-1} \sum_{i=1}^{n-1} \sum_{j=i+1}^{n}\left|a_{i}-a_{j}\right|
\end{aligned}
$$

and we are done.

REMARK 2.2. We have $p^{\prime} \geq p$, where $p$ is defined by (2.1). Hence Theorem 2.1 is in fact stronger than Theorem $C$, since

$$
\left|a_{i}-a_{j}\right| \geq\left(\sqrt{\left|a_{i}\right|}-\sqrt{\left|a_{j}\right|}\right)^{2}
$$


Also, the remark in [1] that the constant factor $p /(n-1)$ cannot be replaced by a greater number is not completely correct. As we see, this factor can be replaced by $p^{\prime} /(n-1)$, which may be greater if $p_{i^{\prime}}=p$.

In fact, we can establish an even better estimate of difference between $\max a_{i}$ and the weighted mean, in terms of the symmetric function $\sum_{i<j}\left|a_{i}-a_{j}\right|$.

Let us prove first following minimax lemma, which may be of independent interest.

LEMMA 2.3. Let $p_{1}, \ldots, p_{n}$ be positive numbers. Then

$$
M:=\sup _{\substack{q_{1}, \ldots, q_{n} \\ q_{1}+\cdots+q_{n}=1}} \min _{\substack{1 \leq i \leq n \\ i \leq j \leq n}} q_{i} p_{j}
$$

is attained and is given by

$$
M=\frac{1}{\sum_{i=1}^{n} 1 / p_{i}^{\prime}},
$$

where $p_{1}^{\prime}, \ldots, p_{n}^{\prime}$ are defined by $p_{i}^{\prime}=\min _{i \leq j \leq n} p_{j}$.

Proof. The minimum of $q_{i} p_{j}$ taken over $1 \leq i \leq n$ and $i \leq j \leq n$ will be nonnegative if and only if each $q_{i}$ is nonnegative, so without loss of generality we may restrict attention to the situation in which $\left\{q_{1}, \ldots, q_{n}\right\}$ is a set of nonnegative probabilities. In this case, that $M$ exists and is attained follows by compactness.

For transparency of reference, the quantities $q_{i} p_{j}$ may be arranged in rows as

$\begin{array}{cccccc}q_{1} p_{1} & q_{1} p_{2} & \cdots & q_{1} p_{i} & \cdots & q_{1} p_{n} \\ & q_{2} p_{2} & \cdots & q_{2} p_{i} & \cdots & q_{2} p_{n} \\ & & & & & \vdots \\ & & & q_{i} p_{i} & \cdots & q_{i} p_{n} \\ & & & & & \vdots \\ & & & & & q_{n} p_{n}\end{array}$.

For each member in the $i$-th row we have $q_{i} p_{j} \geq q_{i} p_{i}^{\prime}$, since $j \geq i$. Suppose that the supremum $M$ is attained for some $n$-tuple $\left(q_{1}, \ldots, q_{n}\right)$ for which $q_{i} p_{i}^{\prime}<q_{j} p_{j}^{\prime}$ for some $i \neq j$. Then we can replace $q_{i}$ by $q_{i}^{\prime}$ and $q_{j}$ by $q_{j}^{\prime}$ such that $q_{i}^{\prime} p_{i}^{\prime}=q_{j}^{\prime} p_{j}^{\prime}$ and $q_{i}^{\prime}+q_{j}^{\prime}=q_{i}+q_{j}$. Doing this will increase the value of the minimum, a contradiction to the definition of $M$.

Hence we have

$$
q_{1} p_{1}^{\prime}=q_{2} p_{2}^{\prime}=\cdots=q_{n} p_{n}^{\prime}=M \text {. }
$$


Since

$$
\sum_{i=1}^{n} \frac{M}{p_{i}^{\prime}}=\sum_{i=1}^{n} q_{i}=1
$$

(2.2) follows.

THEOREM 2.4. Let $p_{i}(i=1, \ldots, n ; n \geq 2)$ be positive real numbers with $\sum_{i=1}^{n} p_{i}=1$ and $a_{1}, \ldots, a_{n}$ real numbers. Let $b_{n} \leq \cdots \leq b_{1}$ be a permutation of $a_{1}, \ldots, a_{n}$, and $r_{1}, \ldots, r_{n}$ the corresponding permutation of $p_{1}, \ldots, p_{n}$. Define

$$
p_{i}^{\prime}=\min _{i \leq j \leq n} r_{j}, \quad i=2, \ldots, n \text {. }
$$

Then

$$
\max _{1 \leq i \leq n} a_{i}-\sum_{i=1}^{n} p_{i} a_{i} \geq \frac{1}{\sum_{i=2}^{n} 1 / p_{i}^{\prime}} \cdot \sum_{1 \leq i<j \leq n}\left|a_{i}-a_{j}\right|
$$

PROOF. For each $i \geq 1$ we have

$$
\max _{1 \leq i \leq n} a_{i}-\sum_{i=1}^{n} p_{i} a_{i}=b_{1}-\sum_{j=1}^{n} r_{j} b_{j}=\sum_{j=2}^{n} r_{j}\left(b_{1}-b_{j}\right) \geq \sum_{j=i+1}^{n} r_{j}\left(b_{i}-b_{j}\right) .
$$

Let $q_{1}, \ldots, q_{n}$ be positive numbers satisfying $q_{1}+\cdots+q_{n}=1$. Multiplying (2.4) by $q_{i}$ and summing over $i=1, \ldots, n-1$ gives

$$
\begin{aligned}
\max _{1 \leq i \leq n} a_{i}-\sum_{i=1}^{n} p_{i} a_{i} & =\frac{1}{1-q_{n}} \sum_{i=1}^{n-1} \sum_{j=2}^{n} q_{i} r_{j}\left(b_{1}-b_{j}\right) \\
& =\sum_{i=1}^{n-1} \sum_{j=2}^{n} q_{i}^{\prime} r_{j}\left(b_{1}-b_{j}\right) \\
& \geq \sum_{i=1}^{n-1} \sum_{j=i+1}^{n} q_{i}^{\prime} r_{j}\left(b_{i}-b_{j}\right),
\end{aligned}
$$

where $q_{i}^{\prime}=q_{i} /\left(1-q_{n}\right)(i=1, \ldots, n-1)$. Since $q_{i}^{\prime}+\cdots+q_{n-1}^{\prime}=1$, Lemma 2.3 yields

$$
\begin{aligned}
\max _{1 \leq i \leq n} a_{i}-\sum_{i=1}^{n} p_{i} a_{i} & \geq \max _{q_{1}, \ldots, q_{n-1}} \min _{1 \leq i<j \leq n} q_{i}^{\prime} r_{j} \sum_{i=1}^{n-1} \sum_{j=i+1}^{n}\left(b_{i}-b_{j}\right) \\
& =\frac{1}{\sum_{i=2}^{n} 1 / p_{i}^{\prime}} \sum_{1 \leq i<j \leq n}\left|a_{i}-a_{j}\right| .
\end{aligned}
$$


REMARK 2.5. Suppose that $a_{n} \leq a_{n-1} \leq \cdots \leq a_{1}$. If $p_{i}^{\prime}=p_{i}$ for all $i \geq 2$, that is, $p_{2} \leq p_{3} \leq \cdots \leq p_{n}$, then the multiplying factor in $(2.3)$ is $\left[1 / p_{2}+\cdots+1 / p_{n}\right]^{-1}$ which can be much better than the value $p_{2} /(n-1)$ in Theorem 2.1 .

COROLlaRY 2.6. For $n=2$, we have

$$
a_{1} p_{1}+a_{2} p_{2}=\max _{i=1,2} a_{i}-p_{2}^{\prime}\left|a_{1}-a_{2}\right|,
$$

where $p_{2}^{\prime}=p_{1}$ if $a_{1}<a_{2}$ and $p_{2}^{\prime}=p_{2}$ if $a_{1}>a_{2}$. In either case, we have

$$
a_{1} p_{1}+a_{2} p_{2} \leq \max _{i=1,2} a_{i}-\min \left(p_{1}, p_{2}\right)\left|a_{1}-a_{2}\right| .
$$

Therefore in the case $n=2,(2.4)$ and (2.3) hold with equality.

\section{Applications}

The improvements to the basic lemma given in the previous section have direct consequences for several theorems given in [17]. The proofs of the first two involve only straightforward modifications of those used in earlier improvements presented in [1] and so are omitted.

THEOREM 3.1. Let $a, b, s_{i}$ and $t_{i}(i=0,1,2)$ be positive real numbers with $a / s_{i}+b / t_{i}=1(i=0,1,2)$ and $s_{1}<s_{0}<s_{2}$, and let $f, g:(0, \infty) \rightarrow \mathbf{R}$ be convex functions. Then

$$
h_{0} \leq \max \left(h_{1}, h_{2}\right)-\alpha\left|h_{1}-h_{2}\right|,
$$

where

$$
h_{i}=\frac{f\left(s_{i}\right)}{s_{i}}+\frac{g\left(t_{i}\right)}{t_{i}} \quad(i=0,1,2)
$$

and

$$
\alpha=\min \left(\frac{\left(s_{2}-s_{0}\right) s_{1}}{\left(s_{2}-s_{1}\right) s_{0}}, \frac{\left(s_{0}-s_{1}\right) s_{2}}{\left(s_{2}-s_{1}\right) s_{0}}\right) \in(0,1) .
$$

Similarly, we have the following for $L^{p}$ norms.

THEOREM 3.2. We adopt the assumptions and notation of the previous theorem.

(i) Suppose the quantities $P_{i}=\|f\|_{s_{i}}\|g\|_{t_{i}}(i=0,1,2)$ all exist. Then

$$
P_{0} \leq \max \left(P_{1}, P_{2}\right)-\alpha\left|P_{1}-P_{2}\right| \text {. }
$$


(ii) Suppose $x=\left(x_{i}\right), u=\left(u_{i}\right)(i=1, \ldots, n)$ and $y=\left(y_{j}\right), v=\left(v_{j}\right)(j=$ $1, \ldots, m)$ are sequences of positive real numbers. Then

$$
Q_{0} \leq \max \left(Q_{1}, Q_{2}\right)-\alpha\left|Q_{1}-Q_{2}\right|
$$

where

$$
\begin{aligned}
Q_{i} & =S_{n}^{\left[s_{i}\right]}(x, u) S_{m}^{\left[t_{i}\right]}(y, v) \quad(i=0,1,2) \quad \text { and } \\
S_{n}^{[t]} & =\left(\sum_{i=1}^{n} u_{i} x_{i}^{t}\right)^{1 / t} .
\end{aligned}
$$

The following result is an improvement of Theorem 1 from [17].

THEOREM 3.3. Suppose that positive numbers $u_{i, j}(i=0,1,2 ; j=1, \ldots, n)$ satisfy $u_{1, j} \geq u_{0, j} \geq u_{2, j}(1 \leq j \leq n)$ and

$$
a_{j} u_{i, 1}+b_{j} u_{i, j}=1 \quad(i=0,1,2 ; 2 \leq j \leq n)
$$

for positive constants $a_{j}, b_{j}(2 \leq j \leq n)$. If $F_{j}:(0, \infty) \rightarrow \mathbf{R}(1 \leq j \leq n)$ are convex functions, then

$$
\sum_{j=1}^{n} F_{j}\left(u_{0, j}\right) \leq \max _{i=1,2}\left\{\sum_{j=1}^{n} F_{j}\left(u_{i, j}\right)\right\}-\min (\lambda, 1-\lambda)\left|\sum_{j=1}^{n}\left[F_{j}\left(u_{1, j}\right)-F_{j}\left(u_{2, j}\right)\right]\right|,
$$

where $\lambda$ is defined by

$$
\lambda:=\frac{u_{2,1}-u_{0,1}}{u_{2,1}-u_{1,1}}
$$

PROOF. From (3.1), we conclude as in Theorem 1 of [17] that

$$
\begin{array}{r}
\lambda=\frac{u_{0, j}-u_{2, j}}{u_{1, j}-u_{2, j}}=\frac{u_{2,1}-u_{0,1}}{u_{2,1}-u_{1,1}}, \\
1-\lambda=\frac{u_{1, j}-u_{0, j}}{u_{1, j}-u_{2, j}}=\frac{u_{0,1}-u_{1,1}}{u_{2,1}-u_{1,1}} .
\end{array}
$$

Also, by the convexity of $F_{j}$,

$$
F_{j}\left(u_{0, j}\right) \leq \frac{u_{0, j}-u_{2, j}}{u_{1, j}-u_{2, j}} F_{j}\left(u_{1, j}\right)+\frac{u_{1, j}-u_{0, j}}{u_{1, j}-u_{2, j}} F_{j}\left(u_{2, j}\right),
$$

that is,

$$
F_{j}\left(u_{0, j}\right) \leq \lambda F_{j}\left(u_{1, j}\right)+(1-\lambda) F_{j}\left(u_{2, j}\right)
$$


After summation, we obtain by Corollary 2.6 that

$$
\begin{aligned}
\sum_{j=1}^{n} F_{j}\left(u_{0, j}\right) & \leq \lambda \sum_{j=1}^{n} F_{j}\left(u_{1, j}\right)+(1-\lambda) \sum_{j=1}^{n} F_{j}\left(u_{2, j}\right) \\
& \leq \max _{i=1,2}\left\{\sum_{j=1}^{n} F_{j}\left(u_{i, j}\right)\right\}-\min (\lambda, 1-\lambda)\left|\sum_{j=1}^{n}\left[F_{j}\left(u_{1, j}\right)-F_{j}\left(u_{2, j}\right)\right]\right| .
\end{aligned}
$$

REMARK 3.4. By Corollary 2.6 , the factor $\min (\lambda, 1-\lambda)$ can be replaced by $\lambda$ or $1-\lambda$, depending on the value of the maximum. The same is true for the factor $\alpha$ in Theorems 3.1 and 3.2.

\section{References}

[1] H. Alzer, "Comments on some inequalities of Pearce and Pečarić", Proc. Edin. Math. Soc. 40 (1997) 167-174.

[2] G. Brown, "Inequalities for measures of sum sets", Proc. CMA ANU 15 (1987) 15-20.

[3] G. Brown, "Some inequalities that arise in measure theory", J. Austral. Math. Soc. Ser. B 45 (1988) 83-94.

[4] G. Brown, M. S. Keane, W. Moran and C. E. M. Pearce, "An inequality with applications to Cantor measure and normal numbers", Mathematika 35 (1988) 87-94.

[5] G. Brown and W. Moran, "Raikov systems and radicals in convolution measure algebras", $J$. London Math. Soc. 28 (1983) 531-542.

[6] G. Brown, C. E. M. Pearce, J. Pečarić and Q. Yin, "Measures of algebraic sums of sets", Math. Ineq. and Applic. 2 (1999) 25-46.

[7] G. Brown and L. A. Shepp, "A convolution inequality", in Contributions to probability and statistics, Essays in honor of Ingram Olkin, (Springer, N. Y., 1989) 51-57.

[8] G. Brown and J. H. Williamson, "Coin tossing and sum sets", J. Austral. Math. Soc. Ser. A 43 (1987) 211-219.

[9] G. Brown and Q. Yin, "Some metric properties of sum sets", in Number theory with an emphasis on the Markoff spectrum, Lecture Notes in Pure and Applied Mathematics 147 (1993) 17-22.

[10] D. Hajela and P. Seymour, "Counting points in hypercubes and convolution measure algebras", Combinatorica 5 (1985) 205-214.

[11] A. W. Kemp, "Certain inequalities involving fractional powers", J. Austral. Math. Soc. Ser. A 53 (1992) 131-136.

[12] H. T. Landau, B. F. Logan and L. A. Shepp, "An inequality conjectured by Hajela and Seymour arising in combinational geometry", Combinatorica 5 (1985) 337-392.

[13] D. M. Oberlin, "The size of sums of sets II", Israel J. Math. 55 (1986) 305-316.

[14] C. E. M. Pearce and J. E. Pečarić, "An inequality for convex functions", J. Math. Anal. Appl. 183 (1994) 523-527.

[15] C. E. M. Pearce and J. E. Pečarić, "On two lemmas of Brown and Shepp having applications to sum sets and fractals", J. Austral. Math. Soc. Ser. B 36 (1994) 60-63.

[16] C. E. M. Pearce and J. E. Pečarić, "On an inequality relating to sum sets", J. Austral. Math. Soc. Ser. B 37 (1995) 208-211. 
[17] C. E. M. Pearce and J. E. Pečarić, "On two lemmas of Brown and Shepp having applications to sum sets and fractals II", J. Austral. Math. Soc Ser. B 37 (1996) 490-494.

[18] A. O. Pittenger, "Inequalities between symmetric, logarithmic and power means", Univ. Beograd Publ. Elektrotechn. Fak. Ser. Mat. Fiz. 678-715 (1980) 19-21.

[19] D. R. Woodall, "A theorem on cubes", Mathematika 24 (1977) 60-62. 